
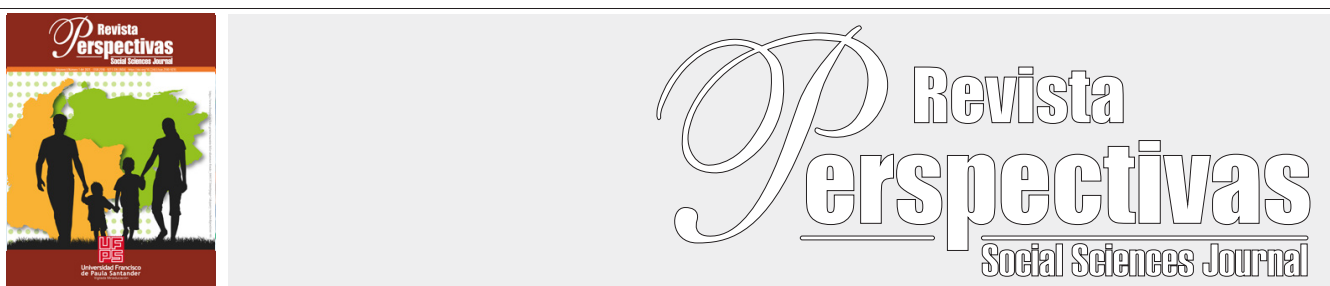

Original Article

https://doi.org/10.22463/25909215.2830

\title{
Uso de la tecnología en el desarrollo de competencias de lectura y de escritura
}

\author{
Use of technology in the development of reading and writing competences
}

Adrián Filiberto Contreras-Colmenares ${ }^{1}$, Imelda Jiménez-Villamarín²,

${ }^{\prime}$ Doctor en Educación, Abogado, Especialista en Derecho Administrativo. Docente Emérito. Profesor Jubilado, categoría Titular de la Universidad de Los Andes-Táchira, Venezuela. Investigador PEI. ULA. Investigador PPI No 6263 (Anterior Programa de Promoción del Investigador en Ciencias Sociales). Correo: adrianfilidi@gmail.com y adriancontreras@ula.ve,ORCID:0000-0001-6711-3649

${ }^{2}$ Licenciada en Educación Básica con Énfasis en Educación Matemática, Especialista en Psicopedagogía Especial, Maestra en Tecnología educativa. Universidad Virtual Escuela de Graduados en Educación. Instituto Tecnológico de Monterrey. México. Magistra en Tecnología Educativa y Medios Innovadores para la Educación. Universidad Autónoma de Bucaramanga. Docente en la Institución Educativa Rural Vijagual, Bucaramanga, Santander, Colombia. imelosfer@ hotmail.com

Como citar: A.F. Contreras-Colmenares, I. Jiménez-Villamarín, M. Martinez-Balaguera “ Uso de la tecnología en el desarrollo de competencias de lectura y de escritura”. Perspectivas, vol. 5, no. 2, pp. 54-71, 2020.

Received: August 01, 2019; Approved: November 19, 2019

\begin{tabular}{ll}
\hline RESUMEN \\
\hline Palabras clave: & $\begin{array}{l}\text { Desde décadas, las Tecnologías de la información y la comunicación (TIC) se conciben como herramientas } \\
\text { favorecedoras de los procesos de enseñanza y aprendizaje. El ser humano con su uso tiene la posibilidad } \\
\text { de aprender y relacionarse. Sin embargo, se hacen evidentes las dificultades de los educandos de primaria }\end{array}$ \\
Tecnologías de & en las competencias comunicativas, que les impiden avanzar en los procesos de lectura y escritura. Por ello, \\
la información y & el propósito del estudio fue identificar el efecto del uso de los recursos tecnológicos en el desarrollo de \\
comunicación, & competencias de lectura y escritura en ambientes de aprendizaje. La metodología seleccionada para realizar \\
competencias, & el estudio fue la cualitativa bajo investigación-acción con apoyo del paradigma socio-crítico. Se tomaron \\
lectura, & 18 participantes de un contexto rural en la Escuela Vijagual. Entre otras, se llegó a la siguiente conclusión: \\
escritura, & Las tareas de lectura y escritura realizadas con uso de TIC fortalecen la interacción entre usuario-máquina \\
habilidades comunicativas. & y motivan al estudiante a realizar procesos de lectura y escritura de manera activa, participativa; en sus \\
& escritos, se evidenció mayor riqueza textual en cuanto a coherencia, sentido y significado.
\end{tabular}

\begin{tabular}{ll}
\hline & ABSTRACT \\
\hline Palabras clave: & $\begin{array}{l}\text { For decades, Information and Communication Technologies (ICT) have been conceived as tools that favor } \\
\text { teaching and learning processes. The human being with its use has the possibility of learning and relating. } \\
\text { Information and }\end{array}$ \\
However, the difficulties of the elementary students in communication skills, which prevent them from \\
advancing in the reading and writing processes, become evident. Therefore, the purpose of the study was \\
technologies, \\
to identify the effect of the use of technological resources in the development of reading and writing skills \\
in learning environments. The methodology selected to carry out the study was the qualitative one under \\
writing, & $\begin{array}{l}\text { research-action supported by the socio-critical paradigm. 18 participants were taken from a rural context in } \\
\text { the Vijagual School. Among others, the following conclusion was reached: The reading and writing tasks } \\
\text { communication abilities. }\end{array}$ \\
& $\begin{array}{l}\text { carried out with the use of ICT strengthen the interaction between user-machine and motivate the student } \\
\text { richness was evidenced in terms of coherence, sense and meaning. }\end{array}$
\end{tabular}

\section{Introducción}

La educación, su estado actual, así como la problemática, se erige en objeto de estudio con un carácter multidisciplinar debido a las disciplinas, desde las cuales puede ser tratada. Igualmente, puede ser multirreferencial y multicontextual en razón de sus diversos escenarios, modalidades y peculiaridades. Tal pluralidad permite, a los investigadores educativos, realizar estudios que puedan comprender dicho fenómeno social $\mathrm{y}$ establecer criterios nuevos que permitan orientar 
la labor docente. De ese modo se puede lograr un equilibrio entre el conocimiento construido y su aplicación en situaciones reales del contexto educativo.Unaperspectiva que adiciona posibilidades de indagación deviene de las exigencias que otorgan las Tecnologías de la Información y la Comunicación (TIC) como elemento esencial en la estructura de la sociedad y también cultural (Adell, 1997).

La incorporación de las TIC puede estar apoyada en el diseño y aplicación de estrategias pedagógicas que permitan tener en el computador el recurso tecnológico que contribuye con la construcción de los procesos de lectura y escritura. Las TIC otorgan una variedad de programas y herramientas que posibilitan el desarrollo de las competencias comunicativas en los diferentes campos de acción de la persona: familiar, social, laboral y educativo, contribuyendo de esta manera a la formación integral de los educandos (García, 2008).

A los efectos de esta revelación investigativa se estableció como objetivo identificar el efecto que tiene el uso de la tecnología en el desarrollo de competencias de lectura y escritura en los estudiantes de primaria de una escuela rural y en ambientes de aprendizaje.

\section{Lo teórico}

En la revisión de la literatura, pudo encontrarse que, en la misma línea de investigación, se han realizado estudios sobre procesos de lectura $\mathrm{y}$ escritura con el uso de herramientas TIC, tanto en el nivel nacional, como en el internacional. Ellos revelan resultados que son tomados como referente para contrastar la información recabada, entre los que se mencionan a nivel nacional, el estudio realizado por Ojeda (2013) menciona en su estudio "El blog educativo y su utilización para estimular las competencias de lectura y escritura", que el uso de las TIC en este caso el edublog, permite estimular y desarrollar las competencias textual, pragmática, semántica y gramatical para mejorar las competencias de lectura y de escritura en los educandos.

Por otro lado, Área Moreira (2009), en su estudio, difiere de las bondades de las TIC por sí solas, porque como lo afirma si las TIC no tienen un planteamiento pedagógico, sencillamente pasarán a ser un elemento más de clase en lugar de ser recursos centrales del aprendizaje. De tal suerte que, como sostiene el mismo Área Moreira (2009, p. 78): ... "la figura del profesor coordinador TIC es un elemento catalizador relevante en el proceso de uso pedagógico de las tecnologías digitales en cada centro escolar".

Otros autores, al respecto, también mencionan que, en los ambientes de aprendizaje, se pueden usar TIC y no alcanzar los aprendizajes esperados; por ello, el efecto de estas herramientas sobre el aprendizaje, depende del uso pedagógico que el docente les dé a esos recursos tecnológicos (Ruiz y Jaramillo, 2009).

Por su parte, Aldana (2012), en su estudio, pudo comprobar cómo el podcast fortalece las competencias de lectura y escritura en niños de primer grado de básica primaria, pues les permite, tanto a los docentes, como a los mismos niños, identificar los avances que cada uno logra progresivamente en la escalera de la psicogénesis de estos dos procesos. Según el autor, el problema radica en que algunos docentes son apáticos al uso de TIC por lo que prefieren aplicar metodologías tradicionales, -mejor, sería denominarlas convencionales-. De igual manera, Padilla y Pedreros (2007) afirman que un docente actualizado y con suficientes conocimientos, será capaz de incorporar recursos e innovar para lograr aprendizajes más significativos.

De igual manera, otro aspecto que favorece la incorporación de las TIC, como recurso o herramienta para promover el aprendizaje, es el grado de interactividad que se logra fortalecer con una mayor intervención entre usuario-máquina; de ese modo, se logra una participación activa, desde el punto de vista comunicacional, pues propicia una adecuada participación del usuario en los entornos 
virtuales. Lo anterior exige una revisión minuciosa, por parte de los mediadores, en torno a este tipo de herramientas antes de ser utilizadas en los procesos de enseñanza aprendizaje, dado que, si los aprendices no han desarrollado las habilidades requeridas para su uso, lo que se obtiene finalmente es un fracaso (Minguell, 2002).

Además, el docente ha de desarrollar, a su vez competencias en el aspecto tecnológico. Como señalan Contreras-Colmenares y Roa-Contreras (2018, p. 1):

Las competencias tecnológicas para el docente, en su desempeño en el contexto educativo, cobra, en la actualidad, más vigencia e importancia, puesto que su dominio e incorporación en el desarrollo del acto pedagógico, le permitirá coadyuvar con la interactuación de sus estudiantes.

Significa la gran responsabilidad que tiene el docente, de autoformarse, para así poder ayudar a los otros, a los aprendices, en la construcción de conocimientos. Mucho más en lo que respecta al aprendizaje de la lectura y la escritura.

A nivel internacional, investigadores de la Universidad de Stanford, con la implementación del proyecto "CAI (computer assisted instruction)" ejecutaron aplicaciones informáticas para apoyar la enseñanza de la lectura y la escritura. Los estudiantes realizaban actividades relacionadas con: ... "identificación de las letras, vocabulario visual, reconocimiento de patrones ortográficos, aprendizaje léxico y comprensión de frases", según Henao (2006, p.73). El computador registraba el desempeño de cada alumno. Los resultados permitieron ratificar que quienes usaron el programa obtuvieron mejores resultados en comparación con quienes utilizaron métodos tradicionales. Es por ello, que como refieren Contreras-Colmenares y Garcés Díaz (2019, p. 215): ... "las Tecnologías de la Información y la Comunicación (TIC) han venido transformando la educación, puesto que, como recurso didáctico, plantean nuevos escenarios, que requieren una revisión profunda del contexto educativo"
Finalmente, Cuevas y Vives (2005) aluden que unas competencias cognitivas que favorecen la capacidad de evaluar un texto que se pueden mencionar son las siguientes: recuperación de la información, interpretación, reflexión y evaluación de dicha información de modo que permita producir nuevo conocimiento. Se valora que, para poder alcanzar tal cometido, son necesarios los procesos de lectura y de escritura, los cuales han de ser apoyados con la incorporación de las TIC. Ello con el propósito de que el educando pueda realizar la búsqueda de la información, así como su uso de manera acertada y significativa.

\section{Método}

La investigación realizada se fundamenta en la orientación cualitativa, en cuanto se desarrolló en un contexto natural, con el propósito de describir las particularidades en relación con los procesos de lectura y escritura, con apoyo en las TIC. Alos efectos se elaboró un proyecto sobre lectura y escritura, con especificidad del contenido relacionado con los géneros literarios en su entorno social, en este caso la escuela. La investigación se basó en la perspectiva de Investigación-Acción (IA) y tuvo como sustento epistemológico el paradigma interpretativo-socio crítico, cuyo principal objetivo es transformar la realidad, es decir que se orientó al cambio educativo a partir de la actividad reflexiva y a la transformación social. (Rodríguez y Valldeoriola, 2009).

El procedimiento fue el siguiente: El tema fue seleccionado a partir de la experiencia investigativa en el área de la lectura y la escritura. Se desarrolló en un contexto rural, dado que las falencias presentadas en las competencias de lectura y escritura, por parte de los educandos afecta su desempeño en las pruebas que se aplican en el área de lenguaje a nivel nacional e internacional, pero, también tiene su impacto en la evaluación de las diferentes áreas del plan de estudio; los resultados obtenidos pueden considerarse como veraces y reales en función de lo metodológico, tal como lo señalan Quecedo y Castaño (2002). 


\section{Contexto}

El contexto, en primer lugar, se ubica en el medio rural. El espacio académico la Institución Educativa Rural Vijagual (IERV), cuya sede principal está ubicada en el kilómetro 10, Vía al Mar, municipio Bucaramanga, Santander. Tiene como representante legal, a quien era el Rector, para la fecha en que se realizó la investigación, el especialista Armando Luna Benavides. Es una institución oficial (pública), de carácter mixta en todos los niveles. La modalidad académica se funda en el Modelo Pedagógico Escuela Nueva - Escuela Activa.

La IERV cuenta con 9 sedes, incluida la sede principal; en ésta funciona primaria en la jornada de la mañana, mientras que en la tarde se atiende bachillerato. La sede tomada como contexto de la investigación, corresponde a la "E” San Ignacio; está ubicada en el kilómetro 11 vía al mar, en la vereda que lleva su mismo nombre. Cuenta con: 3 aulas, una de las cuales funciona como sala de informática, servicios sanitarios, un patio y un restaurante escolar. La matrícula total de la sede es de 50 estudiantes, 29 varones y 21 niñas, quienes proceden de las veredas aledañas a la sede E: La vega, Puente tierra, La sabana y San Ignacio. Las condiciones rurales, que son parte de la comunidad, hacen que algunos alumnos lleguen en moto o carro, pero la mayoría, usualmente, llega a pie.

La jornada de trabajo está establecida de la siguiente manera:

Pre escolar: 6:30 a. m. a 11:00 a. m.

Primaria: 6:30 a. m. a 11:30 m

Secundaria: $12: 00 \mathrm{~m}$. a 6:00 p. m.

\section{Participantes de la investigación}

Los participantes fueron seleccionados bajo el criterio de oportunidad o conveniencia. Como señalan Hernández Sampieri, Fernández y Baptista (2005): ... "simplemente casos disponibles a los cuales tenemos acceso" (p. 401); en total 18 estudiantes de los grados tercero, cuarto y quinto de educación básica primaria. Ellos fueron los alumnos, que, de acuerdo con el interés investigativo, se consideraron los más idóneos, oportunos y convenientes por el contacto permanente que se tenía con ellos, al momento de realizar la actividad indagatoria.

\section{Instrumentos}

Para el estudio se utilizaron como instrumentos de recolección de datos un test de desempeño académico (también conocido como test de educación) relacionado con las competencias en lectura y escritura, cuyas respuestas, proporcionadas por los mismos alumnos, se plantearon mediante las opciones Siempre, A Veces y Nunca. Asimismo, se utilizó el diario de campo, dentro de observación participante y un registro de información, a partir de la observación de documentos.

El test de educación (conocido también como test de desempeño académico) se aplicó en dos momentos del estudio, inicialmente como diagnóstico para identificar pre-saberes con relación a las competencias con que contaban los educandos sobre lectura, escritura y el manejo de los recursos relacionados con las TIC. En un segundo momento, al final después de haber aplicado el proyecto basado en problemas y haber realizado diferentes prácticas con el propósito de identificar los avances obtenidos por los educandos.

Hay que indicar que la observación participante está orientada a tomar en cuenta los comportamientos que solo pueden ser observados y entendidos cuando ocurren de manera natural, Valenzuela y Flórez (2011). Así entonces, durante las diferentes prácticas realizadas se observaron comportamientos, habilidades e interacción en el trabajo colaborativo y cooperativo de los grupos; se registraron, simultáneamente en un diario de campo los datos identificados con relación al uso del lenguaje escrito, verbal, no verbal; así como las prácticas de lectura y 
de escritura en recursos interactivos disponibles en la Web.

En lo que se refiere a los documentos: durante las prácticas los estudiantes realizaron escritos en el procesador de texto Microsoft Word sobre las clases de géneros literarios consultados en le web y realizaron ejemplos de su propia creación, que conformaron un archivo titulado "Mis creaciones". En ellos fue posible observar aspectos, con apoyo en las herramientas TIC, tales como: la comprensión, la interpretación, la reflexión y la evaluación de textos; así como la realización del proceso lector, de la interpretación lectora y del análisis realizado a las lecturas, pero, sobre todo, la comprensión de la lectura.

\section{Procedimiento}

Durante el desarrollo de la investigación se siguieron las cuatro fases de la investigación acción, enfoque que orientó el estudio: fase de planificación, la fase de actividad (acción), fase de recopilación de datos (observación) y la fase de reflexión.

Fase de planificación: Esta fase parte del planteamiento que hace Kemmis (citado por Rodríguez, Herráiz, Prieto, Martínez, Picazo, Castro y Bernal, 2010, p. 19), con base en los siguientes interrogantes: “¿Qué está sucediendo ahora? ¿En qué sentido es problemático? ¿Qué puedo hacer al respecto?"

Las respuestas fueron las siguientes, según la pregunta formulada:

¿Qué está sucediendo ahora?

Los estudiantes presentan dificultades de comprensión lectora y pobre producción textual.

¿En qué sentido es problemático?

Afecta el desempeño de los estudiantes en sus procesos comunicativos, desempeño académico y pruebas saber.

¿Qué puedo hacer al respecto?

Facilitar acciones y estrategias educativas que impliquen el uso de las TIC para que el estudiante tenga la oportunidad de consultar información, así como realizar actividades con recursos interactivos de aprendizaje en la web y ejecutar las asignaciones relacionadas con la lectura y escritura, de modo que les permita fortalecer y mejorar estas habilidades comunicativas y que el docente pueda tener la visión de lo que ocurre con estos procesos, basados en el uso de las tecnologías.

También se realizó la etapa de inmersión que hizo posible identificar en el contexto, en este caso, en la IERV, espacio donde quien investiga se desempeña como docente y los investigados fueron sus mismos estudiantes. Ahora bien, con apego al código de ética del investigador, se solicitó permiso al rector para realizar el estudio por medio de la carta de autorización. De igual manera, en asamblea se hizo lectura de la carta de autorización de padres para que con su firma expresaran su consentimiento para la participación de los niños y niñas, por ser menores de edad, en la realización del estudio.

Fase de actividad (acción): En este tipo de estudio la función de la investigación es servir a la acción; por ello, a partir del diagnóstico realizado (test de desempeño académico), se propuso realizar un proyecto basado en problemas en el que se integró el trabajo activo y colaborativo.

Entre las actividades que se realizaron, se detallan: Consultar en la web sobre los géneros literarios Narrativo (cuento y fábula); lírico (copla, adivinanza y retahíla); dramático (el teatro); concepto y reglas para su elaboración. Crear recursos literarios de su propia autoría; actividad que se realizó en equipos de trabajo. Crear un archivo en Microsoft Word titulado: "Mis creaciones literarias"; realizar redacción de textos; hacer exposiciones, visualización de videos y resolución de test de comprensión lectora.

Para verificar los avances y logros de los educandos durante el desarrollo de las diversas actividades, se aplicó el test de desempeño 
académico, también conocido como test educativo, que hizo posible medir los efectos del plan de acción.

Fase de recopilación: se registraron, de manera minuciosay detallada, las observaciones en un formato de diario de campo que consta de dos partes una de identificación y otra de anotaciones descriptivas e interpretativas. Se transcribió la información, de manera grupal e individual, que permitió clasificar los datos de acuerdo con las categorías: lectura, escritura y manejo de herramientas TIC (computador e Internet) que dieron respuesta a la pregunta de investigación.

Fase de reflexión: Se analizó la información obtenida. En ese sentido, se organizó la información, se codificó y se categorizó para hacerla manejable $\mathrm{y}$, así, facilitar la lectura detallada de los reportes. Finalmente, se estructuró la información analizada en tablas, acompañadas de una explicación detallada.

\section{Organización de la información y el análisis co- rrespondiente}

Los datos recolectados fueron analizados detenidamente a partir de las variables detectadas en la pregunta de investigación lectura, escritura y herramientas TIC, citas de apoyo y los temas o categorías competencia textual, pragmática y semántica. De ahí, que el análisis de los resultados estuviera determinado por los instrumentos aplicados, expuestos a continuación.

Test de desempeño académico o test de educación se aplicó como parte de la primera fase, denominada diagnóstico. La Tabla I muestra las respuestas dadas por los estudiantes con relación a cada categoría, competencia o habilidad planteada según las opciones de respuesta: Siempre, A veces, Nunca.
Tabla I. Resultados del diagnóstico, mediante el test de educación.

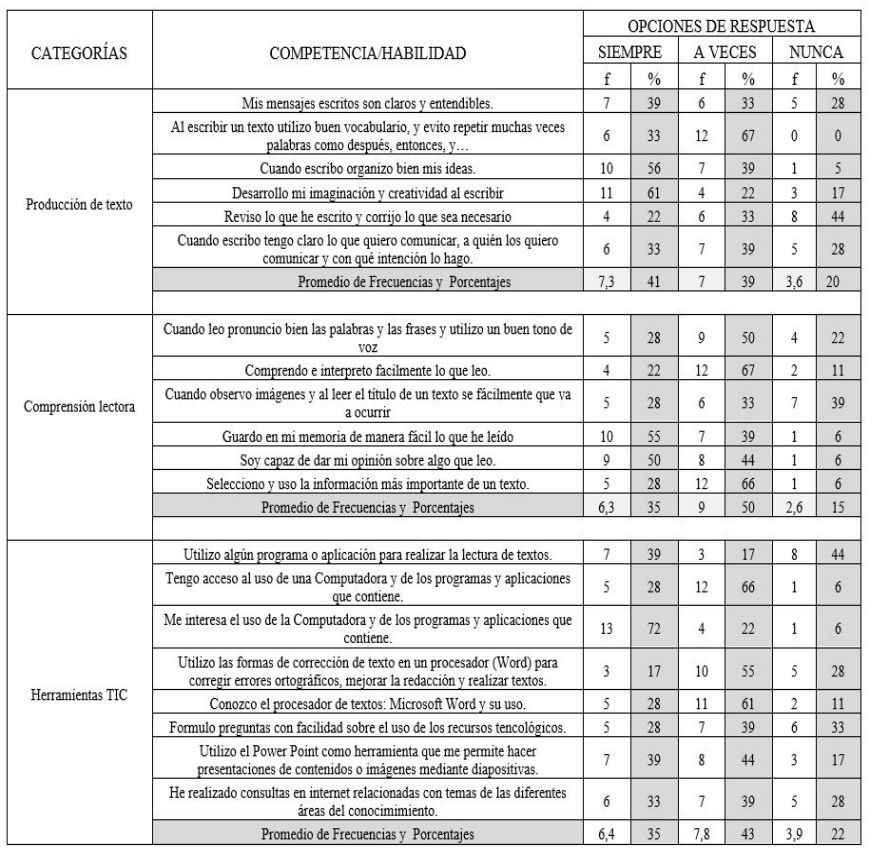

Los resultados de las respuestas dadas por 18 estudiantes a las preguntas presentadas en el test de educación, de acuerdo con las categorías establecidas: producción de texto, comprensión lectora y uso de herramientas TIC según indicadores establecidos: Siempre (S), a veces (AV) o nunca (N).

Se puede visualizar respecto de la categoría producción de texto el $41 \%$ de los estudiantes siempre escriben textos claros, con uso de buen vocabulario; evitan la repetición de palabras, organizan bien las ideas, desarrollan la imaginación y creatividad al escribir; los estudiantes revisan los escritos y corrigen lo necesario, y tienen claro lo que quieren comunicar, a quién lo quieren comunicar y cuál es la intención con que escriben. Mientras que un $39 \%$ cumplen a veces con criterios establecidos para la producción de texto y un $20 \%$, nunca los toman en cuenta.

Al unir las dos opciones de respuestas que se alejan del deber ser, como son: A veces, y Nunca, el porcentaje suma $59 \%$, con lo cual se puede colegir que la producción de texto, hay necesidad de fortalecerla en el espacio escolar. 
Con relación a la categoría comprensión lectora, el 35\% siempre lee con buena entonación y tono de voz, comprende e interpreta con facilidad lo que leen; al leer el título infiere lo que va a ocurrir, guarda en la memoria, opina y selecciona la información más importante de lo que lee. Preocupa que el $50 \%$ a veces cumple con estas competencias, y el $15 \%$, nunca las pone en práctica. Ahora bien, el alejamiento del deber ser, cuando se suma el $50 \%$ de la opción a veces y el $15 \%$ de nunca, se obtiene un $65 \%$, que informa de la debilidad en la comprensión lectora; por ello, hay necesidad de propender a realizar acciones que procuren un mejoramiento en la comprensión lectora de los estudiantes de IERV.

Sobre la categoría herramientas TIC, un $35 \%$ siempre tiene acceso a un computador; vale señalar, tiene mayor contacto con ellas y se interesan por utilizarlas. Por ejemplo, usan el procesador de texto Microsoft Word, la herramienta de creación de presentaciones Power Point; preguntan si no entienden su uso e informan que han realizado consultas en internet. Sin embargo, llama la atención que el $43 \%$ a veces aprovecha las ventajas que proporcionas estas técnicas de Informática y la Comunicación. Así como que el 22\% nunca las utiliza. En esta categoría la conjunción de las dos opciones: a veces y nunca revelan un porcentaje de $65 \%$ que se distancia del deber ser en cuanto al uso, por parte de los estudiantes de los recursos que proporcionan las TIC. Por tanto, se debe realizar una promoción entre los estudiantes, para hacerles ver las ventajas que proporcionan las TIC, para alcanzar un mejor aprendizaje.

A continuación, se incluye la información que devino de la aplicación de la observación. Lo dado se revela de forma descriptiva. Se acompaña lo descrito con una tabla que contiene actividad realizada, el código del estudiante, la etiqueta creada para dar cuenta de lo observado durante la investigación y la cita de apoyo, para cada etiqueta.
De inmediato la descripción: Durante el proyecto basado en problemas se conformaron seis grupos de ocho estudiantes cada uno; ello con el objetivo de promover el trabajo colaborativo y fortalecer el espíritu investigativo. Cada grupo realizó en total doce prácticas entre los meses de mayo y agosto del año 2014, en las que se incluyeron actividades de consulta en Internet sobre cada uno de los géneros literarios narrativo, lírico y dramático (conceptos, características, estructura y elementos), leían la información y, en un archivo creado en Microsoft Word, por cada grupo, redactaban los textos según su capacidad de comprensión y entendimiento. Luego, escribían textos literarios inventados por ellos mismos. Durante la comunicación y ejecución del proyecto se observó en los educandos actitudes de interés y expectativa por el trabajo que realizaban. Como se podrá ver son variadas las etiquetas, que van desde la preocupación hasta el interés, la alegría que conduce a un aprendizaje, basado en el proceso de interacción. Asumir, a su vez, una intención y procurar su uso.

Tabla II.. presentada a continuación muestra las reacciones de los estudiantes.

\begin{tabular}{|c|c|c|c|}
\hline ACTIVDAD & $\begin{array}{l}\text { CÓDIGO DEL } \\
\text { ESTUDIANTE }\end{array}$ & ETIQUETA & CITA DE APOYO \\
\hline \multirow{7}{*}{ Presentacion proyecto } & 14 & Alegria & ¡Qué chévere profe vamos a utilizar bastante los computadores! \\
\hline & 18 & Interés & ¿Vamos autilizar el teclado? \\
\hline & 4 & Preocupación & Pero yo no escribo rápido \\
\hline & 5 & Interaccion & No se preocupe, entre todos podemos ayudar a escribir, no pass nada \\
\hline & 3 & Aprendizaje & Para eso es, parr aprender más a manejar el tecclado \\
\hline & 9 & Uso & $\begin{array}{l}\text { Pero también vamos a utilizar el internet... para encontrar información y } \\
\text { leer otras cosas }\end{array}$ \\
\hline & 11 & Intención & ¡Profet también vamos a jugar! \\
\hline
\end{tabular}

Presentada esta tabla, puede decirse que, en cada práctica realizada, se fue registrando, mediante el diario de campo, las actitudes, comportamientos, así como las reacciones que tuvieron los estudiantes mientras participaban en la realización de las actividades, de acuerdo con los roles que le fueron asignadas por la docente. En lo expuesto, se revela: alegría, interés, así como preocupación. También se logró el proceso de interacción, con lo cual se llegó al aprendizaje de los estudiantes.

En la Tabla III se registran las citas de apoyo expresadas por los estudiantes, en algunas de las 
prácticas, con respecto a los géneros literarios, específicamente, el cuento, las coplas y las adivinanzas, como expresiones del género narrativo.

Tabla III. Registro de citas de apoyo muestra relacionadas con los géneros literarios.

\begin{tabular}{|c|c|c|c|c|}
\hline $\begin{array}{l}\text { FECHA DE LA } \\
\text { PRÁCTICA }\end{array}$ & EQUIPO & TEMA & $\begin{array}{l}\text { CÓDIGO DEL } \\
\text { ESTUDIANTE }\end{array}$ & CITA DE APOYO \\
\hline \multirow{12}{*}{ Mayo 5} & \multirow{12}{*}{ Equipo 1} & \multirow{12}{*}{$\begin{array}{l}\text { Géneros literarios: } \\
\text { género narrativo (el } \\
\text { cuento) }\end{array}$} & Estudiante 6 & 9 prenda el computador \\
\hline & & & Estudiante 13 & Pero espere para saber qué vamos a hacer \\
\hline & & & Estudiante 9 & esperemos qué dice la profesora \\
\hline & & & Estudiante 13 & Si, primero debemos saber quién es el líder \\
\hline & & & Estudiante 6 & Usted 13 \\
\hline & & & Estudiante 13 & bueno y la secretaria entonces es 9 \\
\hline & & & Estudiante 9 & Y... ¿qué va hacer 6 ? \\
\hline & & & Estudiante 13 & Ayudar a leer y a decir lo que vamos a escribir \\
\hline & & & Estudiante 6 & Pero ustedes también me ayudan, todos debemos leer $y$ decir cosas \\
\hline & & & Estudiante 13 & A ver 9 empiece a escribir género literarios. \\
\hline & & & Estudiante 6 & Pero es el narrativo \\
\hline & & & Estudiante 13 & Escuchen para que me ayuden a pensar lo que vamos a escribir \\
\hline \multirow{12}{*}{ Mayo 19} & \multirow{12}{*}{ Equipo 4} & \multirow{12}{*}{$\begin{array}{l}\text { Género lirico: } \\
\text { coplas y } \\
\text { adivinanzas }\end{array}$} & Estudiante 10 & Miren ya encontré el género lirico \\
\hline & & & Estudiante 18 & Muestre a ver qué dice \\
\hline & & & Estudiante 10 & Haber 3 , ayúdenos a leer \\
\hline & & & Estudiante 3 & $\begin{array}{l}\text { Dice: "Este grupo está formado por todas aquellas obras en que el } \\
\text { autor habla de sus sentimientos, refiriéndose a su intimidad" } \\
\text { http://www.portaleducativo.net terra/co }\end{array}$ \\
\hline & & & Estudiante 18 & No entiendo muy bien \\
\hline & & & Estudiante 18 & Mire también salen imágenes, profe podemos buscar ahí. \\
\hline & & & Investigador & $\begin{array}{l}\text { Claro, dan clic sobre el dibujo y sale información que pueden leer } \\
\text { también }\end{array}$ \\
\hline & & & Estudiante 10 & 18 ahí en ese dibujo, espiche \\
\hline & & & Estudiante 3 & $\begin{array}{l}\text { Dice: } L \text { a lirica es el género literario en el que el autor expresa (...) sus } \\
\text { sentimientos y emociones ... }\end{array}$ \\
\hline & & & Estudiante 18 & También dice que se escribe en verso \\
\hline & & & Estudiante 10 & Como las coplas, las adivinanzas y los poemas \\
\hline & & & Estudiante 3 & 10 empiece a escribir antes de que se nos olvide \\
\hline
\end{tabular}

Lo expuesto en la Tabla III evidencia el trabajo realizado de manera conjunta en el que se facilitó la comunicación estudiante-estudiante, estudiante tutor (investigador) y estudiante-contenidos, Hernández y Muñoz (2012). De ahí que los educandos hayan tenido la oportunidad de interactuar directamente con el computador y hacer uso del Internet; de igual manera, se vivenció la interacción entre pares y la orientación del investigador.
En cuanto a la experiencia de usar el procesador de texto Microsoft Word causó expectativa en los estudiantes, pues aplicaron tamaño de fuente, sombreado, cambio de mayúsculas a minúsculas, diseño de página, corrector de ortografía entre otras. Las expresiones de asombro, alegría, sorpresa y el querer saber cada vez más, caracterizaron estas actividades. Algunas de las reacciones de los 
estudiantes al aplicar forma al documento, aparecen en la Tabla IV.

Tabla IV. Reacciones de estudiantes al utilizar el procesador de texto

\begin{tabular}{|c|c|c|c|}
\hline ACTIVIDAD & $\begin{array}{l}\text { CÓDIGO DEL } \\
\text { ESTUDIANTE }\end{array}$ & ETIQUETA & CITA DE APOYO \\
\hline \multirow{12}{*}{$\begin{array}{l}\text { Uso del procesador de } \\
\text { texto: Microsoft Word }\end{array}$} & 17 & Tipo de fuente & Uy hay muchas para escoger... ¿Podemos utilizar la que queramos, profe? \\
\hline & 8 & Tamaño de fuentes & No, así queda muy grande, toca más pequeña \\
\hline & 1 & Color de fuente & A mim ime gusta el color verde \\
\hline & 7 & Color de resaltado de testo & Entonces, podemos ponerle color a los títulos para que se vean mejor... \\
\hline & 12 & Alineación de texto & También podemos dejar los titulos en la mitad... \\
\hline & 17 & $\begin{array}{l}\text { Cambiar mayśscula a } \\
\text { minuscula }\end{array}$ & Eso iba en mayíscula, toca cambiarlo ... \\
\hline & 4 & Vinîtas & Podemos colocarle chulitos a las partes del cuento para que se vea bonito... \\
\hline & 5 & Numeración & Mejor números para que se vea el orden del cuento... \\
\hline & 14 & Color de página & Sí escojamos un color para la hoja, miren cómo le quedo al equipo $4 . .$. \\
\hline & 12 & Borde de página & $\begin{array}{l}\text { Para el orillo de la hoja hay yarias figuras... me gustan los peces... pero los } \\
\text { soles también se ven bonitos... }\end{array}$ \\
\hline & 8 & Insertar figuras pre diseñadas & Para inventar el cuenta busquemos animales... \\
\hline & 4 & Puntración & Profe, ¿cómo puedo poner los dos puntos?... \\
\hline
\end{tabular}

De manera paralela a las sesiones prácticas y enriquecimiento del documento, se observaron los escritos que hacía cada equipo de trabajo para evaluar las competencias: textual, pragmática y semántica para la lectura y la escritura, tal como se muestra en la Tabla V. Ello se hace, a partir de las observaciones de los documentos. En la evaluación se utilizó la escala de valoración establecida para el Sistema Institucional de Evaluación de Estudiantes (SIEE), según decreto 1290 expedido por MEN de Colombia, que en su Artículo 5 menciona: la escala de valoración en las instituciones educativas (IE) debe ser equivalente con los siguientes desempeños: superior $(\mathrm{S})$, alto $(\mathrm{A})$, básico $(\mathrm{BS})$ y bajo $(\mathrm{BJ})$. El valor cuantitativo de cada desempeño equivale a: $\mathrm{S}=4.6-5.0, \quad \mathrm{~A}=4.0-4.5$, BS 3.0-3,9 y $\mathrm{BJ}=1.0-2.9$. (Colombia. Ministerio de Educación Nacional, 2009).

A partir de las abreviaturas establecidas, se presentan los resultados del desempeño de los estudiantes respecto de cada competencia. 
Tabla V. Registro de evaluación competencias: textual, pragmática y semántica

\begin{tabular}{|c|c|c|c|c|c|c|c|}
\hline \multicolumn{2}{|r|}{ COMPETENCIAS } & \multicolumn{6}{|c|}{ EQUIPOS Y LOS DESEMPEÑOS } \\
\hline \multirow{7}{*}{ Lectura } & & 1 & 2 & 3 & 4 & 5 & 6 \\
\hline & Textual & & & & & & A \\
\hline & Comprendo lo que leo & $\mathrm{S}$ & A & A & $\mathrm{S}$ & A & $\mathrm{S}$ \\
\hline & Identifico la intención de acuerdo al tipo de texto (narrativo, lírico o dramático) & $\mathrm{S}$ & $\mathrm{S}$ & A & $\mathrm{S}$ & A & A \\
\hline & Cuestiono las ideas expuestas y las relaciono con otras & $\mathrm{S}$ & $\mathrm{S}$ & A & $\mathrm{S}$ & A & A \\
\hline & Valoro de manera critica lo leído & A & $\mathrm{S}$ & $\mathrm{S}$ & $\mathrm{S}$ & A & A \\
\hline & Reconozco la intención comunicativa del texto & $\mathrm{S}$ & $\mathrm{S}$ & $\mathrm{S}$ & $\mathrm{S}$ & A & A \\
\hline \multirow{4}{*}{ Escritura } & Sé lo que quiero comunicar y la manera como lo debo comunicar & A & A & A & A & A & A \\
\hline & Asumo las implicaciones de lo que digo o escribo & A & $\mathrm{S}$ & A & A & A & A \\
\hline & Autoevalúo lo que he escrito y corrijo lo que sea necesario & $\mathrm{S}$ & $\mathrm{S}$ & A & $\mathrm{S}$ & A & A \\
\hline & Expreso con claridad lo que quiero comunicar & $\mathrm{S}$ & $\mathrm{S}$ & $\mathrm{S}$ & $\mathrm{S}$ & A & A \\
\hline & Pragmática & & & & & & A \\
\hline \multirow{3}{*}{ Lectura } & Leo con Fluidez verbal y precisión las ideas teniendo en cuenta los signos de puntuación & A & A & A & A & A & A \\
\hline & Expreso con claridad lo que comprendo de diferentes tipos de textos & A & $\mathrm{S}$ & A & $\mathrm{S}$ & A & A \\
\hline & Respeto el tumo de la palabra y tengo en cuenta las ideas de los demás & $\mathrm{S}$ & $\mathrm{S}$ & A & $\mathrm{S}$ & A & A \\
\hline \multirow{3}{*}{ Escritura } & Escribo textos de manera organizada & $\mathrm{S}$ & A & A & $\mathrm{S}$ & A & A \\
\hline & Mis escritos presentan ideas con cohesión y coherencia & A & A & $\mathrm{A}$ & $\mathrm{S}$ & BS & A \\
\hline & Al escribir utilizo diversos tipos de sigo, una estructura y secuencia lógica & A & A & A & $\mathrm{S}$ & A & A \\
\hline & Semántica & & & & & & A \\
\hline \multirow{5}{*}{ Lectura } & Expreso hechos de textos leidos siguiendo una secuencia & $\mathrm{S}$ & $\mathrm{S}$ & $\mathrm{S}$ & $\mathrm{S}$ & A & A \\
\hline & Interpreto la intencionalidad del autor y de los personajes & $\mathrm{S}$ & A & A & $\mathrm{S}$ & A & A \\
\hline & Extraigo ideas principales y secundarias y establece relaciones entre ellas & BS & A & BS & A & BS & A \\
\hline & Infiero significados & A & A & A & A & A & A \\
\hline & Interpreto sentimientos e intencionalidades & $\mathrm{S}$ & $\mathrm{S}$ & $\mathrm{S}$ & $\mathrm{S}$ & A & A \\
\hline \multirow{4}{*}{ Escritura } & Escribo textos narrativos, líricos y dramáticos siguiendo una secuencia & $\mathrm{S}$ & A & $\mathrm{S}$ & A & A & A \\
\hline & Tengo clara la intencionalidad de las ideas que expresa en mis escritos & A & A & A & A & A & A \\
\hline & Evidenció riqueza de vocabulario en mis escritos & A & A & A & A & A & A \\
\hline & $\begin{array}{l}\text { Realizo producciones escritas atendiendo a aspectos gramaticales de concordancia, tiempos } \\
\text { verbales, pronombres, sinonimia, antonimia y reglas ortográficas. }\end{array}$ & A & A & A & A & BS & BS \\
\hline
\end{tabular}

De la Tabla V, en torno a la competencia textual, consideradas las dos áreas del lenguaje -lectura y escritura-, puede señalarse lo siguiente: Los equipos 1, 2 y 4, la letra predominante es la letra $\mathrm{S}$, indicativo de un desempeño Superior $(\mathrm{S})$ en esta competencia. En cambio, en los equipos 3, 5 y 6, la letra predominante es A. Con ello se precisa que su desempeño en la competencia es Alto (A). La letra promedio, para esta competencia textual, es la letra A, con lo cual puede aseverarse que los estudiantes, integrantes de los seis (6) equipos, con los cuales se desarrolló la actividad, tienen, en torno a la competencia textual, un desempeño muy favorable.

Ahora, en referencia con la competencia pragmática, tomadas en cuenta, igualmente, las dos áreas lingüísticas -lectura y escritura-, el nivel de desempeño también como puede observarse la letra predominante es la letra $\mathrm{A}$, en los equipos 1 y 2, indicativo de un desempeño Alto (A). En los 
integrantes del equipo 4, se denota un desempeño, en esta competencia específica, valorado como Superior (S), mientras que los integrantes de los equipos 3 y 6 su desempeño se valora como Alto (A). En cambio, en el equipo 5, sus integrantes muestran un desempeño Alto (A), pero en lo que respecta al criterio: "Mis escritos presentan ideas con cohesión y coherencia", su desempeño se ubica en Básico (Bs), por cual ha de seguirse promoviendo, con diferentes actividades, el logro de esta competencia pragmática, muy necesaria en el contexto de la educación, en primer término, y, para su desempeño en todos los actos de lectura y escritura, en segundo término.

En lo que atañe a la competencia semántica, puede referirse que, respecto de las dos áreas lingüísticas -lectura y escritura-, los integrantes del equipo 1, presentan igualdad en cuanto a los desempeños Superior (S) y Alto (A) con la presencia de un desempeño Básico (Bs). En los integrantes del equipo 2 , se denota un predominio del desempeñoAlto (A). Por su parte, en el equipo 3, hay predominio del desempeño Alto (A), con la presencia de desempeño Superior (S), pero también aparece un desempeño Básico (Bs). En lo que respecta a los integrantes del equipo 4, hay predominio del desempeño Alto (A). Con respecto a los integrantes del equipo 5, predomina el desempeño Alto (A), pero aparece, en dos criterios de esta competencia, un desempeño Básico (Bs)

En lo que respecta al equipo 6 hay predominio del desempeño Alto (A), con la presencia de un desempeño Básico (Bs), en el criterio: "Realizo producciones escritas atendiendo a aspectos gramaticales de concordancia, tiempos verbales, pronombres, sinonimia, antonimia y reglas ortográficas". No obstante, llama la atención que en el criterio: "Extraigo ideas principales y secundarias y establece relaciones entre ellas", los integrantes de los equipos 1, 3 y 5, tuvieron un desempeño Básico (Bs). Con ello se plantea la necesidad de propiciar medios y situaciones didácticas que contribuyan a elevar el nivel de desempeño en la competencia semántica.

En el progreso de la investigación, con el propósito de evaluar las competencias comprensión lectora y análisis de texto de manera individual, los estudiantes observaron dos videos. El primero sobre la fábula: "El león y el ratón" y el segundo sobre el cuento: "El leñador honrado". A partir de esta experiencia, se observaron algunos comportamientos, mencionados en la Tabla VI.

Tabla VI. Reacciones de estudiantes durante el visionado de videos

\begin{tabular}{|c|c|}
\hline $\begin{array}{c}\text { CODDIGO DEL } \\
\text { ESTUDIANTE }\end{array}$ & CITA DE APOYO \\
\hline 13 & 3 córnase para yo poder ver bien \\
\hline 3 & Pero si me corro yo tampoco puedo ver \\
\hline 4 & Toca que los de delante de agachen un poco para que los de atrás también veamos. \\
\hline 13 & Al observar el primer vídeo: Es una fábula \\
\hline 5 & No, es un cuento... \\
\hline 11 & Que no 5, porque los personajes son animales... \\
\hline 13 & Eso se llama personificación y la enseñanza de la fábula es una moraleja \\
\hline 10 & Al observa el segundo video: Ese si es un cuento... \\
\hline 18 & Si porque actian personas \\
\hline 13 & En lo del cuento decia que casis siempre los personajes eran personas \\
\hline 3 & Y en este cuento los personajes son la Ninfa y el leñador \\
\hline 1 & Tambièn el vecino del leñador \\
\hline 16 & Los cuentos también dejan una enseñanza \\
\hline 13 & La enseñanza es que debemos ser honestos \\
\hline 10 & Si somos honestos nos va bien \\
\hline 18 & \\
\hline
\end{tabular}

Las intervenciones de los estudiantes, transcritas en la cita de apoyo de la Tabla VI, muestran los conocimientos adquiridos por los estudiantes durante el proyecto basado en problemas lo que hace sus percepciones válidas. Cada estudiante recibió un test de lectura con ítems que evaluaban el nivel de comprensión lectora y análisis de texto.

La Tabla VII muestra algunos ítems respondidos por los estudiantes en los test implementados. En ella se encuentran algunas preguntas con las opciones de respuestas y el número que aparece refiere la cantidad de estudiantes que las seleccionaron, con su respectivo porcentaje. 
Tabla VII. Ítems evaluados en el test de comprensión lectora

\begin{tabular}{|c|c|c|c|c|c|c|c|}
\hline \multirow{3}{*}{ CATEGORÍAS } & \multirow{3}{*}{ COMPETENCIA/HABILIDAD } & \multicolumn{6}{|c|}{ INDICADORES } \\
\hline & & \multicolumn{2}{|c|}{ SIEMPRE } & \multicolumn{2}{|c|}{ A VECES } & \multicolumn{2}{|c|}{ NUNCA } \\
\hline & & $\mathrm{f}$ & $\%$ & $\mathrm{f}$ & $\%$ & $\mathrm{f}$ & $\%$ \\
\hline \multirow{7}{*}{ Producción de texto } & Mis mensajes escritos son claros y entendibles. & 11 & 61 & 7 & 39 & 0 & 0 \\
\hline & $\begin{array}{l}\text { Al escribir un texto utilizo buen vocabulario, y evito repetir muchas } \\
\text { veces palabras como después, entonces, y... }\end{array}$ & 5 & 28 & 12 & 67 & 1 & 6 \\
\hline & Cuando escribo organizo bien mis ideas. & 10 & 56 & 8 & 44 & 0 & 0 \\
\hline & Desarrollo mi imaginación y creatividad al escribir & 15 & 83 & 3 & 17 & 0 & 0 \\
\hline & Reviso lo que he escrito y corrijo lo que sea necesario & 9 & 50 & 7 & 39 & 2 & 11 \\
\hline & $\begin{array}{l}\text { Cuando escribo tengo claro lo que quiero comunicar, a quién los quiero } \\
\text { comunicar y con qué intención lo hago. }\end{array}$ & 16 & 89 & 2 & 11 & 0 & 0 \\
\hline & Promedios de frecuencias y porcentajes & 11 & 61 & 6.5 & 36.1 & 0.5 & 2.8 \\
\hline \multirow{7}{*}{$\begin{array}{l}\text { Comprensión } \\
\text { lectora }\end{array}$} & $\begin{array}{l}\text { Cuando leo pronuncio bien las palabras y las frases y utilizo un buen tono } \\
\text { de voz }\end{array}$ & 6 & 33 & 12 & 67 & 0 & 0 \\
\hline & Comprendo e interpreto facilmente lo que leo. & 8 & 44 & 10 & 56 & 0 & 0 \\
\hline & $\begin{array}{l}\text { Cuando observo imágenes y al leer el título de un texto se fácilmente que } \\
\text { va a ocurrir }\end{array}$ & 11 & 61 & 5 & 28 & 2 & 11 \\
\hline & Guardo en mi memoria de manera fácil lo que he leído & 9 & 50 & 9 & 50 & 0 & 0 \\
\hline & Soy capaz de dar mi opinión sobre algo que leo. & 12 & 67 & 6 & 33 & 0 & 0 \\
\hline & Selecciono y uso la información más importante de un texto. & 10 & 56 & 8 & 44 & 0 & 0 \\
\hline & Promedios de frecuencias y porcentajes & 9.3 & 52 & 8.3 & 46 & 0.3 & 1.9 \\
\hline \multirow{9}{*}{ Herramientas TIC } & Utilizo algún programa o aplicación para realizar la lectura de textos. & 3 & 17 & 15 & 83 & 0 & 0 \\
\hline & $\begin{array}{c}\text { Tengo acceso al uso de una Computadora y de los programas y } \\
\text { aplicaciones que contiene. }\end{array}$ & 7 & 39 & 11 & 61 & 0 & 0 \\
\hline & $\begin{array}{c}\text { Me interesa el uso de la Computadora y de los programas y aplicaciones } \\
\text { que contiene. }\end{array}$ & 16 & 89 & 2 & 11 & 0 & 0 \\
\hline & $\begin{array}{l}\text { Utilizo las formas de corrección de texto en un procesador (Word) para } \\
\text { corregir errores ortográficos, mejorar la redacción y realizar textos. }\end{array}$ & 3 & 17 & 15 & 83 & 0 & 0 \\
\hline & Conozco el procesador de textos: Microsoft Word y su uso. & 4 & 22 & 14 & 78 & 0 & 0 \\
\hline & $\begin{array}{l}\text { Formulo preguntas con facilidad sobre el uso de los recursos } \\
\text { tencológicos. }\end{array}$ & 7 & 39 & 11 & 61 & 0 & 0 \\
\hline & $\begin{array}{l}\text { Utilizo el Power Point como herramienta que me permite hacer } \\
\text { presentaciones de contenidos o imágenes mediante diapositivas. }\end{array}$ & 4 & 22 & 14 & 78 & 0 & 0 \\
\hline & $\begin{array}{l}\text { He realizado consultas en internet relacionadas con temas de las } \\
\text { diferentes áreas del conocimimiento. }\end{array}$ & 10 & 56 & 8 & 45 & 0 & 0 \\
\hline & Promedio de frecuencias y porcentaje & 6.7 & 37.2 & 11.3 & 62.7 & 0 & 0 \\
\hline
\end{tabular}

Al analizar el test de lectura y los apartados referidos en la Tabla VII, y con base en las respuestas de los estudiantes, se hace evidente que los investigados presentan un desempeño significativo en las competencias: semántica, pragmática y textual. Esto evidencia que el trabajo realizado en el proyecto basado en problemas sobre los géneros literarios y la realización de actividades pedagógicas con uso de TIC estimula el desarrollo de las habilidades de lectura y escritura en los educandos.
Prueba de los efectos del plan de acción: Para confirmar si las prácticas realizadas durante el desarrollo del proyecto, fueron efectivas en el fortalecimiento de las habilidades de lectura y de escritura, se aplicó nuevamente el test de educación en esta oportunidad tomado como prueba de los efectos del plan de acción que pretendía evaluar los efectos de las estrategias y prácticas utilizadas durante la etapa de acción, cuyos resultados se muestran en la Tabla VIII. 
Tabla VIII. Resultado prueba de efectos del plan de acción

\begin{tabular}{|c|c|c|c|c|c|c|c|}
\hline \multirow{3}{*}{ CATEGORÍAS } & \multirow{3}{*}{ COMPETENCIA/HABILIDAD } & \multicolumn{6}{|c|}{ INDICADORES } \\
\hline & & \multicolumn{2}{|c|}{ SIEMPRE } & \multicolumn{2}{|c|}{ A VECES } & \multicolumn{2}{|c|}{ NUNCA } \\
\hline & & f & $\%$ & $\mathrm{f}$ & $\%$ & f & $\%$ \\
\hline \multirow{7}{*}{ Producción de texto } & Mis mensajes escritos son claros y entendibles. & 11 & 61 & 7 & 39 & 0 & 0 \\
\hline & $\begin{array}{l}\text { Al escribir un texto utilizo buen vocabulario, y evito repetir muchas } \\
\text { veces palabras como después, entonces, y... }\end{array}$ & 5 & 28 & 12 & 67 & 1 & 6 \\
\hline & Cuando escribo organizo bien mis ideas. & 10 & 56 & 8 & 44 & 0 & 0 \\
\hline & Desarrollo mi imaginación y creatividad al escribir & 15 & 83 & 3 & 17 & 0 & 0 \\
\hline & Reviso lo que he escrito y corrijo lo que sea necesario & 9 & 50 & 7 & 39 & 2 & 11 \\
\hline & $\begin{array}{l}\text { Cuando escribo tengo claro lo que quiero comunicar, a quién los quiero } \\
\text { comunicar y con qué intención lo hago. }\end{array}$ & 16 & 89 & 2 & 11 & 0 & 0 \\
\hline & Promedios de frecuencias y porcentajes & 11 & 61 & 6.5 & 36.1 & 0.5 & 2.8 \\
\hline \multirow{7}{*}{$\begin{array}{l}\text { Comprensión } \\
\text { lectora }\end{array}$} & $\begin{array}{l}\text { Cuando leo pronuncio bien las palabras y las frases y utilizo un buen tono } \\
\text { de voz }\end{array}$ & 6 & 33 & 12 & 67 & 0 & 0 \\
\hline & Comprendo e interpreto facilmente lo que leo. & 8 & 44 & 10 & 56 & 0 & 0 \\
\hline & $\begin{array}{l}\text { Cuando observo imágenes y al leer el título de un texto se fácilmente que } \\
\text { va a ocurrir }\end{array}$ & 11 & 61 & 5 & 28 & 2 & 11 \\
\hline & Guardo en mi memoria de manera fácil lo que he leído & 9 & 50 & 9 & 50 & 0 & 0 \\
\hline & Soy capaz de dar mi opinión sobre algo que leo. & 12 & 67 & 6 & 33 & 0 & 0 \\
\hline & Selecciono y uso la información más importante de un texto. & 10 & 56 & 8 & 44 & 0 & 0 \\
\hline & Promedios de frecuencias y porcentajes & 9.3 & 52 & 8.3 & 46 & 0.3 & 1.9 \\
\hline \multirow{9}{*}{ Herramientas TIC } & Utilizo algún programa o aplicación para realizar la lectura de textos. & 3 & 17 & 15 & 83 & 0 & 0 \\
\hline & $\begin{array}{c}\text { Tengo acceso al uso de una Computadora y de los programas y } \\
\text { aplicaciones que contiene. }\end{array}$ & 7 & 39 & 11 & 61 & 0 & 0 \\
\hline & $\begin{array}{l}\text { Me interesa el uso de la Computadora y de los programas y aplicaciones } \\
\text { que contiene. }\end{array}$ & 16 & 89 & 2 & 11 & 0 & 0 \\
\hline & $\begin{array}{l}\text { Utilizo las formas de corrección de texto en un procesador (Word) para } \\
\text { corregir errores ortográficos, mejorar la redacción y realizar textos. }\end{array}$ & 3 & 17 & 15 & 83 & 0 & 0 \\
\hline & Conozco el procesador de textos: Microsoft Word y su uso. & 4 & 22 & 14 & 78 & 0 & 0 \\
\hline & $\begin{array}{l}\text { Formulo preguntas con facilidad sobre el uso de los recursos } \\
\text { tencologgicos. }\end{array}$ & 7 & 39 & 11 & 61 & 0 & 0 \\
\hline & $\begin{array}{l}\text { Utilizo el Power Point como herramienta que me permite hacer } \\
\text { presentaciones de contenidos o imágenes mediante diapositivas. }\end{array}$ & 4 & 22 & 14 & 78 & 0 & 0 \\
\hline & $\begin{array}{l}\text { He realizado consultas en internet relacionadas con temas de las } \\
\text { diferentes áreas del conocimimiento. }\end{array}$ & 10 & 56 & 8 & 45 & 0 & 0 \\
\hline & Promedio de frecuencias y porcentaje & 6.7 & 37.2 & 11.3 & 62.7 & 0 & 0 \\
\hline
\end{tabular}

La confrontación de los resultados arrojados del diagnóstico y la prueba de los efectos del plan de acción confirman los avances logrados por los educandos en las competencias de lectura y de escritura. Con relación a la categoría Producción de texto, se tiene, en promedio, los siguientes porcentajes: $61 \%$, en la opción siempre; $36 \%$ en la opción A veces, mientras que un $3 \%$ se ubica en la opción Nunca. Se aviene la necesidad de seguir profundizando en el aspecto escritural para que los estudiantes se entusiasmen en producir textos.

En lo que respecta a la categoría Comprensión lectora, de acuerdo con los promedios se revela lo siguiente: el $52 \%$ se ubica en la opción Siempre. En tanto que en la opción A veces, se ubicó el $46 \%$ de las respuestas. Sólo un $2 \%$ se alcanzó en la opción Nunca. Ello permite afirmar que, si se valora la discrepancia respecto del deber ser, hace falta seguir promoviendo la comprensión lectora, mediante la incorporación de estrategias significativas y atractivas.

En cuanto al uso de las herramientas TIC, se observa en promedio en esta categoría que el 37 \% las utiliza Siempre; en cambio, el $63 \%$ se ubicó en la opción A veces. Se denota un alejamiento importante de lo que se aspira a que se ubique en el deber ser, respecto del uso de las herramientas TIC. Por tanto, se requiere que, en la IERV, se impulse, 
con mayor insistencia y vehemencia, el uso de las tecnologías, como apoyo en el proceso de aprendizaje de los estudiantes.

En la Tabla IX se muestra la situación en que se encontraban los estudiantes al aplicar el instrumento en la etapa inicial y final de la investigación.

Tabla IX. Cuadro comparativo diagnóstico- efectos del plan de acción

\begin{tabular}{|c|c|c|c|c|c|c|c|c|c|c|c|c|}
\hline \multirow{3}{*}{ VARIABLES } & \multicolumn{6}{|c|}{ INDICADORES/DIAGNÓSTICO } & \multicolumn{6}{|c|}{$\begin{array}{l}\text { INDICADORES/PRUEBA DE LOS EFECTOS PLAI } \\
\text { DE ACCIÓN }\end{array}$} \\
\hline & \multicolumn{2}{|c|}{ SIEMPRE } & \multicolumn{2}{|c|}{ A VECES } & \multicolumn{2}{|c|}{ NUNCA } & \multicolumn{2}{|c|}{ SIEMPRE } & \multicolumn{2}{|c|}{ A VECES } & \multicolumn{2}{|c|}{ NUNCA } \\
\hline & f & $\%$ & $\mathrm{f}$ & $\%$ & $f$ & $\%$ & F & $\%$ & $\mathrm{f}$ & $\%$ & $\mathrm{f}$ & $\%$ \\
\hline \multirow{6}{*}{$\begin{array}{l}\text { Producción de texto. } \\
\text { Competencias } 1 \text { a } 6\end{array}$} & 7 & 39 & 6 & 33 & 5 & 28 & 11 & 61 & 7 & 39 & 0 & 0 \\
\hline & 6 & 33 & 12 & 67 & 0 & 0 & 5 & 28 & 12 & 67 & 1 & 6 \\
\hline & 10 & 56 & 7 & 39 & 1 & 6 & 10 & 56 & 8 & 44 & 0 & 0 \\
\hline & 11 & 61 & 4 & 22 & 3 & 17 & 15 & 83 & 3 & 17 & 0 & 0 \\
\hline & 4 & 22 & 6 & 33 & 8 & 44 & 9 & 50 & 7 & 39 & 2 & 11 \\
\hline & 6 & 33 & 7 & 39 & 5 & 28 & 16 & 89 & 2 & 11 & 0 & 0 \\
\hline Promedio & 7.3 & 41 & 7 & 39 & 3.6 & 20 & 11 & 61 & 6.5 & 36 & 0.5 & 3 \\
\hline \multirow{6}{*}{$\begin{array}{c}\text { Comprensión } \\
\text { lectora. } \\
\text { Competencias } 7 \text { a } 12\end{array}$} & 5 & 28 & 9 & 50 & 4 & 22 & 6 & 33 & 12 & 67 & 0 & 0 \\
\hline & 4 & 22 & 12 & 67 & 2 & 11 & 8 & 44 & 10 & 56 & 0 & 0 \\
\hline & 5 & 28 & 6 & 33 & 7 & 39 & 11 & 61 & 5 & 28 & 2 & 11 \\
\hline & 10 & 56 & 7 & 39 & 1 & 6 & 9 & 50 & 9 & 50 & 0 & 0 \\
\hline & 9 & 50 & 8 & 44 & 1 & 6 & 12 & 67 & 6 & 33 & 0 & 0 \\
\hline & 5 & 28 & 12 & 67 & 1 & 6 & 10 & 56 & 8 & 44 & 0 & 0 \\
\hline Promedio & 6.3 & 35 & 9 & 50 & 2.6 & 15 & 9.3 & 52 & 8.3 & 46 & 0.3 & 2 \\
\hline \multirow{8}{*}{$\begin{array}{l}\text { Herramientas TIC. } \\
\text { Competencias } 13 \mathrm{a} \\
20\end{array}$} & 7 & 39 & 3 & 17 & 8 & 44 & 3 & 17 & 15 & 83 & 0 & 0 \\
\hline & 5 & 28 & 12 & 67 & 1 & 6 & 7 & 39 & 11 & 61 & 0 & 0 \\
\hline & 13 & 72 & 4 & 22 & 1 & 6 & 16 & 89 & 2 & 11 & 0 & 0 \\
\hline & 3 & 17 & 10 & 56 & 5 & 28 & 3 & 17 & 15 & 83 & 0 & 0 \\
\hline & 5 & 28 & 11 & 61 & 2 & 11 & 4 & 22 & 14 & 78 & 0 & 0 \\
\hline & 4 & 22 & 7 & 39 & 6 & 33 & 7 & 39 & 11 & 61 & 0 & 0 \\
\hline & 7 & 39 & 8 & 44 & 3 & 17 & 4 & 22 & 14 & 78 & 0 & 0 \\
\hline & 6 & 33 & 7 & 39 & 5 & 28 & 10 & 56 & 8 & 44 & 0 & 0 \\
\hline Promedio & 6.2 & 35 & 7.8 & 43 & 3.9 & 22 & 6.8 & 38 & 11.2 & 62 & 0 & 0 \\
\hline
\end{tabular}


En la Tabla IX, se observan los resultados obtenidos, en cada una de las variables siguientes: producción de textos, comprensión lectora y uso de las herramientas TIC, tanto del diagnóstico, como de los efectos de la aplicación del plan de acción. En ese sentido, en el diagnóstico, en la variable producción de textos el resultado fue el siguiente: $33 \%$ en la opción Siempre; 39 \% en la opción A veces; y 28 $\%$ en la categoría Nunca. Con la aplicación del plan de acción se denota un mejoramiento importante, pues los resultados son los siguientes: $89 \%$ en la opción Siempre, mientras que $11 \%$ en la opción A veces. De modo que puede colegirse que el plan de acción en lo que respecta a la producción de textos ha resultado efectivo, aun cuando hay que seguir desarrollando, hasta alcanzar el ideal que es el 100 $\%$ en la opción Siempre.

Respecto de la variable Comprensión lectora, los resultados del diagnóstico en contraste con los efectos del plan de acción para alcanzar un mejoramiento se tiene los siguientes resultados: 35 $\%$ en la opción Siempre; 50 \% en la opción A veces; y $15 \%$ en la opción Nunca. Con la aplicación del plan de acción se alcanzaron los siguientes resultados: 52 $\%$ en la opción Siempre, mientras que $46 \%$ en la opción A veces y $2 \%$ en la opción Nunca. Si bien hubo una mejora sustancial, aún es necesario seguir profundizando con aplicación de nuevos planes de acción para alcanzar una mayor y mejor comprensión lectora en los estudiantes de la IERV.

En relación con la variable Herramientas TIC, los resultados del diagnóstico son los siguientes: $35 \%$ en la opción Siempre; $43 \%$ en la opción A veces; y $22 \%$ en la opción Nunca. Con la aplicación del plan de acción se alcanzaron los siguientes resultados: $38 \%$ en la opción Siempre, mientras que $46 \%$ en la opción A veces y en la opción Nunca no hubo valor porcentual. Una explicación posible de no haberse dado un mejor resultado está en que los estudiantes solamente tienen acceso a un computador durante la jornada escolar; por lo que se concluye que este aspecto marcó, en gran manera la diferencia observada, con relación al uso y manejo de las herramientas TIC.

\section{Discusión}

Las categorías de análisis seleccionadas a partir de la pregunta de investigación producción de texto, comprensión lectora y manejo de herramientas TIC contribuyeron a dar respuesta a la pregunta que orientó el estudio ¿De qué manera el uso de TIC apoya el desarrollo de competencias de lectura y de escritura en los estudiantes de primaria de una Institución Educativa del sector rural colombiano? En función de ella, se pudo demostrar, con el diagnóstico, en qué nivel se encontraban los estudiantes al inicio del estudio. Luego, se evidencia, cómo se fortalecieron las competencias textual, pragmática y semántica con las diversas estrategias pedagógicas incorporadas al aprendizaje durante el desarrollo del proyecto, basado en problemas. Se informa, además, sobre los avances logrados según resultados del test aplicado para corroborar los efectos de del plan de acción, con lo cual se logra superar, considerablemente, las falencias detectadas en los procesos de lectura y escritura.

A partir de la pregunta de investigación: ¿De qué manera el uso de TIC apoya el desarrollo de competencias de lectura y de escritura en los estudiantes de primaria de una Institución Educativa del sector rural colombiano? y de acuerdo a los datos y resultados ya mencionados, se comprobó cómo el diseño y desarrollo de tareas escolares, con uso de las TIC, promueven el desarrollo de competencias de lectura y escritura en los educandos; en este caso, a partir de la aplicación del proyecto de aprendizaje basado en problemas, permitió evidenciar avances significativos de los estudiantes en los procesos comunicativos mencionados. Un progreso fue la interacción de los educandos, con las herramientas TIC durante el desarrollo del proyecto, en actividades de lectura, escritura, búsqueda y análisis de información sobre los géneros literarios y la producción de textos narrativos, líricos y dramáticos; también en el análisis de vídeos, desarrollo de 
test sobre comprensión lectora con procesos de pensamiento antes, durante y después de lo visionado facilitaron, a los estudiantes, experiencias que, de manera práctica y progresiva, fueron fortaleciendo sus habilidades de lectura y de escritura, así como el desarrollo de las competencias textual, pragmática y semántica.

Loanteriorpermite dar respuesta a lomanifestado por Ojeda (2013) en su investigación, cuando afirma que el uso de TIC, en su caso el edublog, permite estimular y desarrollar las competencias textual, pragmática, semántica y gramatical para mejorar procesos de lectura y escritura en los educandos. Una posición distinta es la de Área Moreira (2009) quien afirma que la mayoría de veces el uso de tecnologías no tiene un planteamiento didáctico, cuando se incluye como un elemento más de la clase y no como un recurso de apoyo al aprendizaje.

No se puede generalizar este hallazgo si se tiene en cuenta que las estrategias pedagógicas planteadas fueron planteadas por el docente las que determinaron el efecto de las TIC en el aprendizaje. Se toma como fundamento de lo expresado, la teoría sociocultural de Vygotsky, (citado por Contreras-Colmenares, 2004) quien considera a la escuela como el espacio de interacción del estudiante con el docente y sus compañeros, con lo cual se puede alcanzar la Zona de Desarrollo Próximo. Por ello, el trabajo realizado en equipo, la asignación de roles, las planeación y organización de tareas, la participación activa y continua, el diseño de productos que evidencien el trabajo elaborado por ellos mismos y que al ser sustentados les permitan demostrar los aprendizajes construidos con el apoyo de sus pares y del docente. Esta situación fue observada durante las prácticas Vygotsky (expuesta por Carrera y Mazzarella, 2001).

Se quiso constatar el logro del objetivo general planteado: "Identificar el efecto del uso de tecnología en el desarrollo de competencias de lectura y de escritura en estudiantes de los grados tercero, cuarto y quinto de la institución educativa Rural Vijagual para afianzar el desempeño de los educandos en los procesos comunicativos de su entorno sociocultural".

Para ello, durante el desarrollo de la investigación y la realización de las diferentes actividades escolares, se hizo uso de las TIC (computador, procesador de texto, Internet, vídeos y recursos interactivos de aprendizaje) en el proyecto basado en problemas, y puede, sobre esa base, señalar que los educandos sí lograron fortalecer las competencias de lenguaje. Competencias concretadas en: búsqueda de información, comprensión del lenguaje, intencionalidad y tipo de texto, comunicación de ideas para tomar decisiones y llegar a acuerdos, comprensión de mensajes orales y escritos, de palabras y frases. Y en lo que compete a la escritura: redacción, coherencia textual, capacidad descriptiva, riqueza de vocabulario, uso de signos de puntuación, ortografía y creatividad, articulación de palabras y frases.

Lo anterior ratifica lo mencionado por los investigadores Reinking y Schreiner, en 1985 y Anderson Inman y Horney, en 1998, (citados por Henao, 2006) quienes indican que con el uso de TIC (textos en pantalla, audio, vídeos, gráficas), se logra mejorar la comprensión lectora en alumnos de quinto grado. Cabe mencionar que esta no es una tarea acabada; por el contrario, hay que seguir fortaleciéndola día a día dado que la lectura y la escritura son procesos que se encuentran inmersos en cada etapa de desarrollo del ser humano.

\section{Conclusiones}

Durante el transcurso del estudio se ha realizado un análisis sobre el efecto del uso de TIC en los procesos de lectura y escritura en las IE, de básica primaria de carácter oficial del municipio de Bucaramanga, específicamente en la Institución Educativa Rural Vijagual.

En primer lugar, se detectó que los estudiantes del sector rural cuentan con pocas habilidades en el uso de herramientas TIC como consecuencia de 
la brecha digital existente entre el sector urbano y rural. Es evidente que, aunque el gobierno ha tratado de minimizar este fenómeno, en ocasiones se quedan solo en buenas intenciones, porque no todos los entes comprometidos asumen las responsabilidades correspondientes. Los resultados del diagnóstico también muestran cómo al inicio de la investigación ellos contaban con bajos niveles de lectura y escritura que se fueron fortaleciendo poco a poco, con las prácticas realizadas durante el proyecto basado en problemas.

De igual manera, es imprescindible que los docentes planteen estrategias pedagógicas con el uso de TIC, orientadas a la formación de los educandos en el manejo y correcto uso de estas herramientas para fortalecer la formación académica de los educandos en las diferentes áreas del conocimiento; estrategias que estén dirigidas, también, a la formación de correctos procesos comunicativos que incluyan el fortalecimiento de la lectura, escritura y oralidad. De modo que para el logro de tal propósito es indispensable el andamiaje del docente en el correcto uso de herramientas TIC, a partir de unas metas, objetivos, actividades, tareas y resultados reales que fortalezcan la formación integral de los educandos.

\section{Referencias}

Adell, J. (1997). Tendencias en educación en la sociedad de las tecnologías de la información. Revista electrónica de tecnología educativa, 7 , EDUTEC. Recuperado de: http://nti.uji.es/docs/ nti/Jordi_Adell_EDUTEC.html

Aldana, T. (2012). El podcast en la enseñanza de la lectoescritura. Red de Revistas Cientificas de América Latina, el Caribe, España y Portugal. Sistema de Información Científica, 37, 9-26. Recuperado de: http://www.redalyc.org/ articuloBasic.oa?id=194224568002

Área Moreira, M. (2010). El proceso de integración y uso pedagógico de las TIC en los centros educativos. Un estudio de casos. Revista de Educación, (352), pp. 77-97. Recuperado de: http://www.revistaeducacion.mec.es/re352/ re352_04.pdf

Carrera, B. y Mazzarella, C. (2001). Vygotsky: enfoque sociocultural. Educere, 5(13), 4244. Recuperado de: http://www.redalyc.org/ pdf/356/35601309.pdf

Contreras-Colmenares, A. (2004). Mediación de procesos cognitivos en el aprendizaje de la lectura. San Cristóbal, Venezuela: Litoformas.

Contreras-Colmenares, A. y Garcés-Díaz, L. (2019). Ambientes Virtuales de Aprendizaje: dificultades de uso en los estudiantes de cuarto grado de primaria. En Revista Prospectiva. Revista de Trabajo Social e intervención social No. 27, enero-junio 2019: pp. 215-240. [Artículo en línea]. Recuperado el 18 de abril de 2019. Recuperado de: http://historiayespacio. univalle.edu.co/index.php/prospectiva/article/ view/7273/9783 y en http://revistas.univalle.edu. co/index.php/prospectiva/article/view/7273

Contreras-Colmenares, A. y Roa-Contreras, M. (2018). Competencias tecnológicas para el desarrollo de contenidos de bioquímica por parte de los docentes. En Revista digital La Pasión del Saber. Año 8, 14 y número 15, año 2019. [Artículo en línea] Recuperado el 18 de abril de 2019. Recuperado de: http://201.249.74.131/pasion/ index.php/95-edicion-14/232-competenciastecnologicas-para-el-desarrollo-de-contenidosde-bioquimica-por-parte-de-los-docentes

Cuevas, C. y Vives. G. (2005). La competencia lectora en el estudio PISA. Un análisis desde la alfabetización en información. Anales de documentación, 8, 51-70. Recuperado de: http:// revistas.um.es/analesdoc/article/view/1541/1601

García, J. L. (2008). Las ciencias de la comunicación 
a la luz de las nuevas tecnologías: retos para una disciplina en la incertidumbre. Red de Revistas Científicas de América Latina, el Caribe, España y Portugal. Sistema de Información Científica, 5(10), 1-15. Recuperado de http:/gmje.mty. itesm.mx/lascienciasdelacomunicacion.pdf

Henao, A. (2006). Evidencias de la investigación sobre el impacto de las tecnologías de información y comunicación en la enseñanza de la lectoescritura. Revista educación pedagógica, 18(44), 73-87. Recuperado de: http://aprendeenlinea. udea.edu.co/revistas/index.php/revistaeyp/ article/viewArticle/6073

Hernández Sampieri, R.; Fernández, C. y Baptista, P. (2005). Metodología de la investigación. México: McGraw-Hill.

Hernández, N. y Muñoz A. (2012). Usos de las tecnologías de la información y la comunicación (TIC) en un proceso formal de enseñanza y aprendizaje en la Educación Básica. Red de Revistas Científicas de América Latina, el Caribe, España y Portugal, (16), 2-13. Recuperado de: http://www.redalyc.org/articulo. oa? $\mathrm{id}=85323935002$

Ojeda, N. (2013). El blog educativo y su utilización para estimular las competencias lectoescritoras. Revista Electrónica de Investigación y Docencia, 8, 38-60.

Padilla, G. y Pedreros, M. (2007). Tecnologías de información y comunicaciones (tic) en el aula del siglo xxi: cómo acompañar a profesores y profesoras en este desafío. Rev. Pensamiento Educativo, 40(1), 391-411. Recuperado de: http:// pensamientoeducativo.uc.cl/files/journals/2/ articles/413/public/413-922-1-PB.pdf.

Quecedo, R. y Castaño, C. (2002). Introducción a la metodología de investigación cualitativa. Revista de Psicodidáctica, (14), 1-24.
Recuperado de: http://www.redalyc.org/articulo. oa? id $=17501402$

Rodríguez, D.; Valldeoriola, J. (2009). Metodología de la investigación. Módulo en línea. Recuperado de: http://openaccess.uoc. edu/webapps/o2/bitstream/10609/77608/2/ Metodolog\%C3\%ADa\%20de\%201a\%20 investigaci\%C3\%B3n_M\%C3\%B3dulo\%201. pdf

Rodríguez, S.; Herráiz, N.; Prieto, M.; Martínez, M.; Picazo, M.; Castro, I. y Bernal, S. (2010). Investigación acción. Obtenida el 12 de marzo de 2014, Recuperado de http://www.uam.es/ personal_pdi/stmaria/jmurillo/InvestigacionEE/ Presentaciones/Curso_10/Inv_accion_trabajo. pdf

Ruiz, M. y Jaramillo, P. (2009). Un caso de integración de TIC que no agrega valor al aprendizaje. Revista latinoamericana de ciencias sociales niñez juventud 7(1), 267-287. Recuperado de: http://revistaumanizales.cinde.org.co/index.php/ Revista-Latinoamericana/article/view/228/111

Valenzuela J. y Flores M. (2011) Fundamentos de la investigación educativa, Volumen 2 y 3. Monterrey, México: Editorial Digital. 